\title{
TAXA DE GESTAÇÃO DE RECEPTORAS DE EMBRIÕES BOVINOS COM DIFERENTES GRAUS DE DIFICULDADES NO PROCEDIMENTO DE INOVULAÇÃO
}

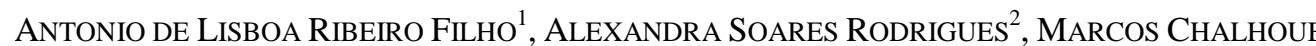 \\ COElHo LimA ${ }^{1}$, Priscila ASSIS FERRAZ ${ }^{2}$, MARCUS ViníCIUS GALVÃo LOIOLA ${ }^{1}$, RODRIGO FreITAS \\ BITTENCOURT $^{3}$

\footnotetext{
${ }^{1}$ Professor Doutor da Universidade Federal da Bahia, Salvador, BA - alisboafilho@ uol.com.br

${ }^{2}$ Graduandos em Medicina Veterinária da Universidade Federal da Bahia, Salvador, BA
} \\ ${ }^{3}$ Professor Colaborador da Universidade Estadual Paulista Júlio de Mesquita Filho, Botucatu, SP.
}

Com o objetivo de avaliar a influência da dificuldade de transposição da cérvix, do tônus uterino e do local de inovulação sobre a taxa de gestação de receptoras de embriões bovinos, realizou-se superovulação e sincronização de doadoras e receptoras. A dificuldade de transposição cervical influenciou a taxa de gestação, o grupo CERV-F (cérvix de fácil transposição) obteve taxa de gestação de 50,60\% (420/830), já CERV-D (com algum grau de dificuldade de transposição) obteve taxa de 43,02\% $(114 / 265)$. O tônus uterino não afetou a taxa de gestação, os grupos TON-R (útero relaxado), TON-M (medianamente relaxado) e TON-C (contraído) tiveram taxas de 50,08\%
$(313 / 625), 45,69 \% \quad(106 / 232)$ e $47,11 \% \quad(106 / 225)$, respectivamente. Foi demonstrado efeito do local de inovulação sobre a taxa de gestação, o grupo DEP-F (inovulação no terço final) obtiveram taxa de gestação de $51,39 \%$ (314/611), superior à taxa de gestação do grupo DEP-I (no terço inicial) que foi de 39,46\% (58/147), já os animais do grupo DEP-M (no terço médio) lograram taxa de gestação de 48,22\% (163/338), não diferindo dos grupos DEP-I e DEP-F. Os resultados sugerem que em programas de transferência de embriões o técnico deverá levar em consideração especialmente a dificuldade de transposição cervical e o local de inovulação.

PALAVRAS-CHAVE: Bos indicus; taxa de prenhez; transferência de embrião.

\section{PREGNANCY RATE OF CATTLE EMBRYO RECIPIENT WITH DIFFERENT DEGREES OF DIFFICULTY ON EMBRYO TRANSFER PROCEEDING}

\section{ABSTRACT}

To evaluate the influence of the difficulty of cervix transposition, uterine tonus and place of embryo trnasfer on pregnancy rate of cattle embryos recipient, the superovulation and synchronization of donor and recipients were carried out. The difficulty of cervix transposition influenced the pregnancy rate. The pregnancy rate was $50.50 \%$ (420/830) for group CERV-E (cervix of easy transposition) and $43.02 \%$ (114/265) for group CERV-D (with some degree of difficulty of transposition). The uterine tonus did not affect the pregnancy rate. The groups TON-R (relaxed uterus), TON-M (medium relaxed uterus) and TON-C (contracted uterus) achieved rates of $50.08 \%$ (313/625), $45.69 \%$ $(106 / 232)$ and $47.11 \%(106 / 225)$, respectively. Data also showed that the embryo transfer place affected the recipient pregnancy rate. Group DEP-F (embryo transfer at the final third) achieved pregnancy rate of $51.39 \%$ (314/611), higher than group DEP-I (at the initial third), which was $39.46 \%$ (58/147), while group DEP-M (at the middle third) achieved pregnancy rate of $48.22 \%$ (163/338), similar to DEP-I and DEP-F groups. The results suggest that, in embryo transfer programs, the technician should take into special account the difficulty of cervix transposition and the embryo transfer place.

KEYWORDS: Bos indicus; embryo transfer; pregnancy rate. 


\section{INTRODUÇÃO}

A transferência de embriões (TE) é uma biotécnica mundialmente difundida, com mais de 500.000 embriões bovinos sendo produzidos e transferidos a cada ano (HASLER, 2003). O Brasil tornou-se referência na área da reprodução animal devido aos avanços e à vasta aplicação dessa técnica, havendo um aumento significativo no número de TEs realizadas no país (VIANA \& CAMARGO, 2007).

Entretanto, a taxa de gestação das receptoras destaca-se como um dos pontos de estrangulamento na difusão dessa tecnologia. Após a transferência de embriões classificados como morfologicamente viáveis, esse índice situa-se em torno de 55\%, o que representa uma elevação no custo unitário de cada produto, impactando negativamente a atividade e influenciando de forma desfavorável o produtor no momento de decidir adotar essa técnica (FERNANDES, 1999).

Vários fatores afetam a taxa de gestação das receptoras como aspectos intrínsecos ao embrião, ao ambiente uterino e, sobretudo, as variáveis relacionadas ao processo de inovulação (SPELL et al., 2001). Nesse contexto, em um procedimento não cirúrgico de inovulação, a receptora é examinada para se identificar o ovário que contém o corpo lúteo (LEAL et al., 2009). Faz-se a passagem do aplicador pela vagina e cérvix e em seguida o embrião é depositado no corno ipslateral ao ovário que contém corpo lúteo (JAINUDEEN et al., 2004). Entretanto, variações morfológicas na cérvix podem aumentar a manipulação do trato genital, possibilitando o aparecimento de lesões (SREENAN \& DISKIN, 1987) ou, dependendo do tônus muscular e do grau de enovelamento dos cornos, haverá uma maior dificuldade de manipulação do útero e até impossibilidade de inovulação no local adequado, o terço final do corno uterino (DIAZ, 1988).

Dessa forma, diferentes graus de dificuldades no procedimento de inovulação podem afetar a taxa de gestação das receptoras (CUTINI et al., 2000). Assim, no momento da realização da técnica o operador deve levar em consideração essas variáveis para que o programa tenha sucesso (SREENAN \& DISKIN, 1987; JLTA,1995; FERNANDES, 1999).

Devido à importância do estudo dessas características, este trabalho teve como objetivo avaliar a influência da dificuldade de transposição da cérvix, do tônus uterino e do local de inovulação sobre a taxa de gestação de receptoras de embriões bovinos.

\section{MATERIAL E MÉTODOS}

$\mathrm{O}$ experimento foi conduzido em diferentes propriedades localizadas na região semi-árida do Estado da Bahia, durante o período de 2007/2008. Foram realizadas 155 colheitas de embriões em doadoras da raça Nelore e foram utilizadas 1.096 novilhas mestiças Bos taurus taurus x Bos taurus indicus como receptoras. Os animais foram mantidos em piquetes de capim Brachiara decumbens, com água e sal mineral ad libitum. As novilhas utilizadas tinham idade variando entre 1,8 a 3,5 anos, pesavam entre 310 a $450 \mathrm{~kg}$ e apresentavam índice de escore corporal entre 2,5 a 3,5 (escala de 1 a 5). Esses animais foram submetidos ao exame clínicoginecológico prévio com o propósito de se certificar a capacidade dos mesmos em responderem ao protocolo de sincronização.

Este trabalho foi aprovado pela Comissão de Ética da Escola de Medicina Veterinária e Zootecnia da Universidade Federal da Bahia, sob o $n^{\circ}$ 25/2011.

Anteriormente à transferência propriamente dita, os animais (doadoras e receptoras) tiveram que passar pelos tratamentos hormonais e procedimentos apresentados a seguir. Em estágio aleatório do ciclo estral, no dia denominado como Dia 0, as doadoras tiveram a onda folicular sincronizada por um dispositivo intravaginal de progesterona (DIB, Shering Plough, São Paulo, Brasil) associado a $2 \mathrm{mg}$ de Benzoato de Estradiol intramuscular (i.m.) (Gonadiol, Shering Plough, São Paulo, Brasil). A superestimulação ovariana foi realizada com 250U.I. de FSH extraído de hipófise suína (Pluset, HertapeCalier, São Paulo, Brasil) em oito doses decrescentes i.m. de 12 em 12 horas, a partir do Dia 4. No Dia 6 pela manhã, administraram-se $500 \mu \mathrm{g}$ de Cloprostenol i.m. (Ciosin, Shering Plough, São Paulo, Brasil). Os dispositivos foram retirados no Dia 7, 24 horas após a aplicação do Cloprostenol. As ovulações foram induzidas com administração de $100 \mu \mathrm{g}$ de Acetato de Fertirelina i.m. (Fertigen, Shering Plough, São Paulo, Brasil), realizadas no Dia 8 pela manhã. As inseminações foram executadas em tempo fixo de 12 e 24 horas após a indução das ovulações, utilizando-se sêmen criopreservado comercializado.

As colheitas de embriões foram efetuadas de 6,5 a 7 dias após a primeira inseminação artificial. Anteriormente a cada colheita, foi procedida a limpeza e assepsia da região perineal das doadoras com água e álcool a 70\%, respectivamente. Também 
foi realizada a anestesia epidural, com o objetivo de diminuir o peristaltismo e desconforto dos animais durante o procedimento. A recuperação dos embriões foi realizada por meio do método não cirúrgico através da lavagem do útero com DPBS (Embriocare, Cultilab, Campinas, Brasil) aquecido a $37^{\circ} \mathrm{C}$. Imediatamente após as colheitas os embriões foram transferidos para uma solução de manutenção e cultura contendo 0,4\% de BSA (Embriocare Solução de Manutenção, Cultilab, Campinas, Brasil), logo após, foram classificados conforme seu estágio de desenvolvimento e qualidade de acordo com os padrões adotados pela Sociedade Internacional de Transferência de Embriões (IETS, 1998), apenas aqueles classificados em estágio de 4 a 7 (mórula, blastocisto inicial, blastocisto e blastocisto expandido, respectivamente) e qualificados entre $1 \mathrm{a}$ 3 (excelente ou bom, regular e pobre) foram envasados em palhetas de $0,25 \mathrm{~mL}$ e transferidos.

A preparação das receptoras para inovulação começou no Dia 0, quando as mesmas receberam um dispositivo intravaginal de progesterona (DIB, Shering Plough, São Paulo, Brasil) associado a $2 \mathrm{mg}$ de Benzoato de Estradiol i.m. (Gonadiol, Shering Plough, São Paulo, Brasil). No Dia 5 foram tratadas com 300U.I. de eCG i.m. (Novormon, Shering Plough, São Paulo, Brasil) e 500 $\mu$ g de Cloprostenol i.m. (Ciosin, Shering Plough, São Paulo, Brasil). No Dia 8 os dispositivos intravaginais foram retirados e no Dia 9 os animais receberam $1 \mathrm{mg}$ de Benzoato de Estradiol i.m. (Gonadiol, Shering Plough, São Paulo, Brasil). Os embriões foram transferidos à fresco no $17^{\circ}$ dia do protocolo de sincronização. No dia da transferência, os ovários das receptoras foram palpados por via retal e examinados por ultrassonografia utilizando-se um aparelho de ultrassom Pie-Medical (Falcon 100, Nutricell, São Paulo, Brasil) com transdutor linear de $5 \mathrm{MHz}$ para se detectar a presença de um corpo lúteo (CL). Receptoras que não tiveram tecido luteal ou um CL com pelo menos $13 \mathrm{~mm}$ foram descartadas. Anteriormente a cada inovulação, os mesmos procedimentos referentes à limpeza, assepsia e anestesia realizados nas doadoras foram conferidos às receptoras.

Os embriões foram transferidos por meio do método não cirúrgico. Imediatamente após as inovulações, as variáveis, dificuldade de transposição da cérvix, tônus do útero e local de inovulação foram avaliadas e anotadas em fichas especialmente confeccionadas para esse fim. Para a classificação da dificuldade de transposição cervical foi considerado o tempo despendido para a passagem completa do aplicador pela cérvix, sendo denomina cérvix de difícil transposição quando o tempo despendido foi igual ou superior a três minutos. $\mathrm{O}$ tônus uterino foi classificado de acordo com o grau de contratilidade dos cornos uterino. Já o local de inovulação foi classificado segundo a posição onde o embrião foi depositado no corno uterino ipsilateral ao corpo lúteo. Essas inovulações e avaliações foram executadas por um único técnico experiente.

Os grupos experimentais foram separados da seguinte forma: dificuldade de transposição da cérvix - CERV-F (n=830), cérvix de fácil transposição (tempo para transposição inferior a três minutos) e CERV-D $(n=265)$, cérvix com algum grau de dificuldade de transposição (tempo para transposição igual ou superior a três minutos); tônus uterino TON-R ( $\mathrm{n}=625)$, útero relaxado, TON-M $(\mathrm{n}=232)$, útero medianamente relaxado (contratilidade intermediária entre útero relaxado e contraído) e TON-C $(n=225)$, útero contraído; local de inovulação - DEP-I $(n=147)$, inovulação no terço inicial do corno uterino, DEP-M $(\mathrm{n}=338)$, inovulação no terço médio do corno uterino e DEP-F ( $\mathrm{n}=611)$, inovulação no terço final do corno uterino.

O diagnóstico de gestação foi realizado aos 35 dias após a inovulação por meio de ultrassonografia transretal, utilizando-se um aparelho de ultrassom Pie-Medical (Falcon 100, Nutricell, São Paulo, Brasil) com transdutor linear de 5,0 MHz.

Para se comparar a taxa de gestação entre as características avaliadas utilizou-se um estudo de dispersão de frequências, empregando-se o teste de qui-quadrado $\left(\chi^{2}\right)$, por meio do pacote estatístico Statistical Analysis System - versão 6 (SAS, 1996).

\section{RESULTADOS E DISCUSSÃO}

Neste experimento verificou-se uma taxa de gestação geral de 48,81\% (535/1.096). A dificuldade de transposição cervical (Tabela 1) influenciou a taxa de gestação $(\mathrm{P}=0,032)$. O Grupo CERV-F obteve taxa de gestação de 50,60\% (420/830), já o Grupo CERV-D obteve taxa de 43,02\% (114/265). Esces resultados corroboram com os obtidos por FERNANDES (1999), que observaram que animais que apresentaram uma maior dificuldade de transposição da cérvix, devido a alterações na disposição dos anéis transversais, tiveram uma maior possibilidade de lesão do mesmo, e consequentemente, menores taxas de gestação.

Esse fato é explicado pelas lesões cervicais, assim como, pelas as manipulações excessivas, as quais podem aumentar as concentrações de prostaglandina $\mathrm{F}_{2} \alpha\left(\mathrm{PGF}_{2} \alpha\right)$ no sangue e no lúmen uterino induzindo a luteólise na vaca, levando à perda embrionária precoce e influenciando negativamente a taxa de gestação das receptoras, como descrito por MCNAUGHTAN (2004). 
Tabela 1 - Taxa de gestação de receptoras classificadas de acordo com a dificuldade de transposição cervical, CERV-F - cérvix de fácil transposição e CERV-D - cérvix com algum grau de dificuldade de transposição

\begin{tabular}{lcc}
\multicolumn{1}{c}{ Grupos experimentais } & Número de animais & $\begin{array}{c}\text { Taxa de Gestação } \\
\mathrm{N}^{\circ}(\%)\end{array}$ \\
\hline CERV-F & 830 & $420(50,60 \%) \mathrm{a}$ \\
CERV-D & 265 & $114(43,02 \%) \mathrm{b}$ \\
\hline Total & 1.095 & $534(48,77 \%)$ \\
\hline
\end{tabular}

Valores seguidos de letras distintas na coluna diferem entre si $(\mathrm{P}=0,032)$ pelo teste de $\chi^{2}$.

O tônus uterino não afetou a taxa de gestação $(\mathrm{P}=0,465)$. Como demonstrado na Tabela 2, os grupos TON-R, TON-M e TON-C tiveram taxas de
$50,08 \%(313 / 625), 45,69 \%(106 / 232)$ e $47,11 \%$ (106/225), respectivamente.

Tabela 2 - Taxa de gestação de receptoras classificadas de acordo com o tônus uterino, TON-R - útero relaxado, TON-M - útero medianamente relaxado e TON-C - útero contraído

\begin{tabular}{lcc}
\multicolumn{1}{c}{ Grupos experimentais } & Número de animais & $\begin{array}{c}\text { Taxa de Gestação } \\
\mathrm{N}^{\circ}(\%)\end{array}$ \\
\hline TON-R & 625 & $313(50,08 \%)$ \\
TON-M & 232 & $106(45,69 \%)$ \\
TON-C & 225 & $106(47,11 \%)$ \\
\hline Total & 1.082 & $525(48,52 \%)$ \\
\hline
\end{tabular}

Esse resultado diverge do que foi reportado por DIAZ (1988) e FERNANDES (1999), que verificaram que receptoras que possuíam uma difícil manipulação uterina, ou seja, maior tônus muscular, apresentaram resultados inferiores em programas de TE. Ainda segundo os mesmos autores, esse fato pode ser justificado pela maior ocorrência de lesões endometriais pelo aplicador, ou mesmo devido ao grau de enovelamento dos cornos e maior dificuldade ou até impossibilidade de inovulação no local adequado, o terço final do corno uterino.

Difere também dos resultados obtidos por LEAL et al. (2009), que observaram que receptoras com útero em estágio intermediário de contratilidade foram as que apresentaram melhores resultados de taxa de gestação em programas de TE, se comparadas às receptoras que apresentaram útero flácido, ou seja, com tônus uterino relaxado. Esses autores ainda sugeriram que as taxas de gestação foram comprometidas porque as excessivas manipulações do útero e as lesões endometriais aumentam os níveis de $\mathrm{PGF}_{2} \alpha$ no sangue e esse aumento pode afetar indiretamente o estabelecimento da prenhez, pois compromete a função luteal, provocando luteólise ao se ligar aos receptores existentes no corpo lúteo, levando a um decréscimo na concentração de progesterona. Além disso, essa prostaglandina pode ter o efeito direto sobre o embrião, interferindo no desenvolvimento embrionário e afetando sua viabilidade.

Por outro lado, segundo SREENAN \& DISKIN (1987), CUTINI et al. (2000) e LOONEY et al., (2006), operadores experientes e consequentemente mais habilidosos tecnicamente tiveram maior facilidade em manobrar os cornos uterinos mais tortuosos e de difícil manipulação; dessa forma, eles conseguiram minimizar as lesões provocadas ao endométrio e obtiveram taxas de gestação semelhantes quando trabalharam com receptoras com diferentes graus de tonicidade uterina. Esses resultados podem justificar a semelhança nas taxas de gestação quando se comparou os grupos TON-R, TON-M e TON-C.

A contratilidade uterina pode também, ser um reflexo dos níveis de progesterona presentes, uma vez que esse hormônio age sobre o endométrio reduzindo sua tonicidade e exercendo um papel luteotrófico, favorecendo a implantação embrionária (BERTAN et al., 2005; LIMA \& SOUZA, 2009). Assim, os melhores índices de gestação para os animais com útero relaxado que foram observados por DIAZ (1988) e FERNANDES (1999) podem ter 
ocorrido também em decorrência dos maiores níveis de progesterona presentes. Entretanto, nesta pesquisa, esse mecanismo não foi observado, sendo sugestiva a realização de estudos futuros incluindo os níveis séricos de progesterona presentes nas receptoras no momento da inovulação.

A análise estatística demonstrou ainda efeito do local de inovulação sobre a taxa de gestação das receptoras (Tabela 3). Os animais do Grupo DEP-F obtiveram taxa de gestação de 51,39\% (314/611), superior $(\mathrm{P}=0,009)$ à taxa de gestação do Grupo DEP-I que foi de 39,46\% (58/147), já os animais do Grupo DEP-M lograram taxa de gestação de 48,22\% (163/338), não diferindo dos Grupos DEP-I $(\mathrm{P}=0,075)$ e DEP-F $(\mathrm{P}=0,350)$.

Em concordância com os resultados obtidos por BEAL et al. (1998), a taxa de gestação foi significativamente maior no grupo em que os embriões foram inovulados mais profundamente no corno uterino (DEP-F: no terço final do corno uterino) em relação ao grupo mais superficial (DEPI: terço inicial do corno uterino). Porém, essa taxa divergiu do estudo de SREENAN \& DISKIN (1987), que sugeriram que a transferência de embriões para depois do terço médio com um cateter rígido pode lesionar o endométrio e levar a uma mortalidade embrionária. Dentro da mesma linha de raciocínio, PIERONI et al. (2008), trabalhando com embriões transferidos à fresco e congelados/descongelados, sugeriram que a inovulação pode ser realizada em qualquer porção do corno uterino adjacente ao ovário contendo o corpo lúteo.

Tabela 3 - Taxa de gestação de receptoras classificadas de acordo com o local de inovulação, DEP-F inovulação no terço final, DEP-M - inovulação no terço médio e DEP-I - inovulação no terço inicial do corno uterino

\begin{tabular}{lcc}
\hline \multicolumn{1}{c}{ Grupos experimentais } & Número de animais & $\begin{array}{c}\text { Taxa de Gestação } \\
\mathrm{N}^{\mathbf{0}}(\%)\end{array}$ \\
\hline DEP-F & 611 & $314(51,39 \%) \mathrm{a}$ \\
DEP-M & 338 & $163(48,22 \%) \mathrm{ab}$ \\
DEP-I & 225 & $58(39,46 \%) \mathrm{b}$ \\
\hline Total & 1.096 & $535(48,81 \%)$ \\
\hline Valores seguidos de letras distintas na coluna diferem entre si (DEP-FxDEP-M: P=0,350; DEP-FxDEP-I: P=0,009; DEP-IxDEP-M: \\
P=0,075) pelo teste de $\chi^{2}$.
\end{tabular}

Não se observou diferença significativa nas taxas de gestação quando os embriões foram inovulados no terço médio do corno uterino (DEP$\mathrm{M})$, em relação àqueles inovulados no terço inicial (DEP-I) e os inovulados no terço final (DEP-F).

Esses resultados podem ser explicados, provavelmente, devido a uma maior proximidade do embrião ao corpo lúteo, sofrendo uma maior ação do fator luteotrófico (progesterona) produzido por ele, assim como, uma maior ação dos sinais antiluteolíticos secretados pelo concepto, que tem o objetivo de prolongar a vida do corpo lúteo e favorecer o reconhecimento materno da gestação e a viabilidade embrionária (MCNAUGHTAN, 2004; LIMA \& SOUZA, 2009).

No entanto, esses benefícios só ocorrem quando a inovulação é feita por um técnico experiente, em que o grau de manipulação e lesão do endométrio é mínimo, pois a inserção forçada do aplicador para partes mais profundas do corno, às vezes, danifica o endométrio podendo causar falhas na transferência, conforme relatado por LOONEY et al., (2006). Assim, quando se têm cornos muito enovelados e de difícil manipulação, é preferível que a inovulação seja feita no terço médio do corno uterino do que em partes mais profundas onde se tem uma grande possibilidade de lesionar endométrio.

$\mathrm{Na}$ literatura, a maioria dos trabalhos consultados utilizaram animais de raças taurinas e os experimentos foram conduzidos em regiões de clima temperado (SREENAN \& DISKIN, 1987; DIAZ, 1988; FERNANDES, 1999), o que difere do presente estudo e pode justificar as divergências encontradas entre os mesmos para algumas variáveis. Entretanto, as particularidades populacionais e regionais deste experimento tornam-o mais fidedigno à realidade da pecuária brasileira, na qual a maioria da população de bovinos são zebuínos criados à pasto em condições tropicais.

\section{CONCLUSÕES}

Os resultados alcançados sugerem que o tônus uterino no momento da inovulação parece não comprometer a taxa de gestação e que o técnico responsável deverá levar em consideração de maneira especial a dificuldade de transposição cervical e o local de inovulação no útero em um 
programa de transferência de embriões, pois essas características foram as que impactaram a taxa de gestação das receptoras. Assim, ao se trabalhar em um programa de transferência de embriões, deve-se fazer a seleção das receptoras que apresentem as melhores condições de inovulação, utilizando-se como parâmetros as características estudadas no presente trabalho, com intuito de aumentar as taxas de gestação.

\section{AGRADECIMENTOS}

Nosso agradecimento à empresa Shering Plough, pelo apoio e doação dos hormônios, à Fundação de Amparo à Pesquisa do Estado da Bahia (FAPESB) e ao Conselho Nacional de Desenvolvimento Cientifico e Tecnológico (CNPq) pelo apoio financeiro.

\section{REFERÊNCIAS}

BEAL, W.E.; HINSHAW, R.H.; WHITMAN, S.S. Evaluating embryo freezing method and the site of embryo deposition on pregnancy rate in bovine embryo transfer. Theriogenology, v.49, n. 1, p.241, 1998.

BERTAN, C.M.; BINELLI, M.; MADUREIRA, E.H.; TRALDI, A.S. Mecanismos Endócrinos e Moleculares envolvidos na formação do corpo lúteo e na luteólise Revisão Literatura. Brazilian Journal of Veterinary Research Animal Science v.43, n.6, p.824-840, 2006.

CUTINI, A.; TERUEL, M.; CABODEVILA, J. Factores que determinan el resultado de la transferencia no quirurgica de embriones bovinos. Revista Taurus, v.2, n. 8, p.35-47, 2000.

DIAZ, A.P. Criopreservação e transferência não cirúrgica de embriões bovinos: alguns aspectos que influenciam a taxa de gestação. 1988. 79f. Dissertação (Mestrado em Medicina Veterinária) - Universidade Federal de Minas Gerais, Belo Horizonte.

FERNANDES, C.A.C. Inovulações não cirúrgicas e taxa de gestação de receptoras de embrião. Arquivo Brasileiro de Medicina Veterinária e Zootecnia, v.51, n. 3, p.263266, 1999.

HASLER, J.F. The current status and future of commercial embryo transfer in cattle. Animal Reproduction Science, v.79, n. 3-4, p.245-264, 2003.

JAINUDEEN, M.R.; WAHID, H.; HAFEZ, E.S.E.
Indução da Ovulação, Produção e Transferência de Embriões. In: HAFEZ, E.S.E.; HAFEZ, B. Reprodução Animal. 7. ed. Rio de Janeiro: Manole, 2004. p.409-434.

JAPAN LIVESTOCK TECHNOLOGY ASSOCIATION - JLTA. Manual of Bovine Embryo Transfer. 1.ed. Tóquio: JLTA, 1995. 432p.

LEAL, L.S.; OBA, E.; FERNANDES, C.A.C.; SÁ FILHO, O.G. Avaliação do corpo lúteo, contratilidade uterina e concentrações plasmáticas de progesterona e estradiol em receptoras de embriões bovinos. Ciência Animal Brasileira, v.10, n.1, p. 174-183, 2009.

LIMA, I.M.T.; SOUZA, A.L. Desenvolvimento e Sobrevivência de Embriões no Período de Préimplantação: Enfoque em Ruminante. Revista Brasileira de Reprodução Animal. v.33, n.4, p.194-202, 2009.

LOONEY, C.R.; NELSON, J.S.; SCHNEIDER, H.J.; FORREST, D.W. Improving fertility in beef cow recipients. Theriogenology, v.65, n. 1, p.201-2009, 2006.

MCNAUGHTAN , J.W. The Effect of Prostaglandin Inhibitor on Pregnancy Rates of Heifer Embryo Transfer Recipients. 2004. 30f. Dissertação (Mestrado em Medicina Veterinária) - Brigham Young University, Provo.Disponível em http://contentdm.lib.byu.edu/ ETD/image/etd672.pdf

PIERONI, J.S.P.; RODRIGUES, C.A.; TEIXEIRA, A.A.; MANCILHA, R.F.; OLIVEIRA, M.E.F.; FERREIRA, R.M.; FRANCESCHINI, P.H. Influência do local de inovulação sobre a taxa de concepção após transferência de embriões frescos e congelados/descongelados produzidos in vivo. Acta Scientiae Veterinariae, v.36, n. 2, p.579, 2008.

SAS Institute Inc. SAS user's guide for Windows Environment: 6.12. Cary, NC, SAS Institute: 1996. 79p.

SOCIEDADE INTERNACIONAL DE TRANSFERÊNCIA DE EMBRIÕES - IETS. Manual da Sociedade Internacional de Transferência de Embriões. 3.ed. Savoy: IETS, 1998. 180p.

SREENAN, J.M.; DISKIN, M.G. Factors affecting pregnancy rates following embryo transfer in the cow. Theriogenology, v.27, n. 1, p.99-113, 1987.

SPELL, A.R.; BELL, W.E.; CORAH, L.R.; LAMB, G.C. Evaluating Recipient and Embryo Factors that affect pregnancy rates of embryo transfer in beef cattle. Theriogenology, v.56, n. 1, p.287-297, 2001.

VIANA, J.H.M.; CAMARGO, L.S.A. A produção de embriões bovinos no Brasil: uma nova realidade. Acta Scientiae Veterinariae, v.35, n. 3, p.915-924, 2007. 